\title{
LA PSICOPEDAGOGÍA COMO DISCIPLINA: UNA PERSPECTIVA CONTEXTUALISTA Y DE CICLO VITAL
}

\author{
PSICOPEDAGOGY AS A DISCIPLINE: \\ AN ENVIRONMENTAL AND LIFE-CYCLE APPROACH
}

\author{
David Poveda* \\ Universidad Autónoma de Madrid
}

\begin{abstract}
RESUMEN
Este artículo plantea aproximarse a la Psicopedagogía desde dos ejes, uno compuesto por los contextos de desarrollo en los que participan los seres humanos y otro compuesto por las diferentes etapas del ciclo vital. Así, la cuestión clave es comprender cómo el desarrollo educativo a lo largo del tiempo esta íntimamente relacionado con diferentes contextos sociales. Para las personas en desarrollo esto implica enfrentarse a las continuidades y discontinuidades entre los contextos y etapas del desarrollo en las que participan. Desde el punto de vista de competencias profesionales este marco implica desarrollar dos tipos de habilidades: (a) la capacidad para establecer puentes entre contextos y profesionales y, (b) desarrollar una visión reflexiva del trabajo educativo. Finalmente, se discute cómo este marco puede servir para interpretar la intervención psicopedagógica en cuatro temas emergentes: la ampliación de la noción de escolarización, el uso de las nuevas tecnologías, la participación comunitaria y la diversidad cultural.
\end{abstract}

Palabras clave: Contexto - Ciclo Vital - Psicopedagogía.

\begin{abstract}
This paper suggests approaching Psychopedagogy from two axis, one made of different developmental contexts and another one made of the different stages of the life-span. In this way, the key consideration is to understand how educational development in the life-course is intimately tied to different social contexts. For the developing person, this implies confronting the continuities and discontinuities between the contexts and life stages in which she or he participates. From the point of view of professional skills this implies developing two kinds of capacities: (a) to be able to build bridges between contexts and professionals and, (b) promoting a reflexive vision of educational work. Finally,
\end{abstract}

\footnotetext{
* David Poveda es profesor en el Departamento de Psicología Educativa y de la Educación de la Universidad Autónoma de Madrid. Sus principales áreas de investigación son la alfabetización, la diversidad social y el discurso en diferentes contextos de desarrollo, todo ello desde una perspectiva etnográfica.
} 
this framework is used to interpret psychopedagogical interventions in four emergent fields: the broadening of schooling, using new technologies of communication, community participation and cultural diversity.

Key words: Context - Life-Span - Psychopedagogy.

\section{Introducción}

A pesar de que existen referencias a la Psicopedagogía como profesión y como disciplina desde hace más de un siglo, en la actualidad la asociación más inmediata de este término es con el ámbito de intervención desarrollado para el sistema educativo formal a través de la LOGSE (y su implantación como titulación universitaria a lo largo de los años 90'). El trabajo en equipos y departamentos de orientación psicopedagógica en los niveles educativos anteriores a la universidad continúa siendo el referente principal de la Psicopedagogía y, probablemente, en cuanto a volumen laboral ocupa todavía el campo de trabajo más importante. Sin duda, la psicopedagogía tiene un papel muy importante dentro del sistema educativo formal y todavía debe desplegar dentro de este ámbito todo su potencial.

Sin embargo, hay múltiples razones para no reducir el campo de reflexión y trabajo de la psicopedagogía al sistema educativo formal e incluso parece necesario contextualizar el papel que desempeña el sistema educativo en el proceso más amplio y prolongado, en el tiempo y el espacio, que consideramos educación. Diversos planes de estudio del la Licenciatura de Psicopedagogía intentan dar respuesta a esta perspectiva amplia al establecer itinerarios escolares y socio-laborales, poniendo de manifiesto que la formación y los procesos educativos, cuando menos, tienen también lugar en el campo social y laboral. El sistema educativo y el sistema laboral son dos de los espacios institucionales más importantes en la vida de muchas personas, tanto por las horas que pasamos en ellos como por el impacto que tienen en nuestras trayectorias vitales y capacidad de movimiento en el sistema social (cf. Rockwell, 1995; Willis, 1993). No obstante, incluso esta oposición entre intervención escolar-intervención laboral puede considerarse una simplificación entre dos espacios prototípicos para la labor psicopedagógica. Entre y alrededor de estos dos ámbitos centrales existen numerosos espacios de intervención y desarrollo educativo muy importantes (la familia, el tiempo libre, la salud, la formación no reglada, etc.) que también deben ser considerados y que no pueden ser asimilados exclusivamente con las herramientas desarrolladas para comprender la intervención educativa en ámbitos escolares y laborales.

Dado este panorama, el objetivo de este trabajo es proporcionar un marco para organizar estos espacios educativos y señalar algunos de los desafíos que puede presentar el trabajo profesional y la investigación dentro de ellos. Para ello, la presentación se va a organizar a lo largo de cuatro puntos. En primer lugar, se presenta una visión anclada en el desarrollo humano de los ámbitos educativos en los que participamos los miembros de nuestra sociedad. En segundo lugar, dado este marco inicial, se presentan algunas cuestiones en torno a los desafíos específicos que presenta el trabajo psicopedagógico $\mathrm{y}$, por tanto, el tipo de competencias y habilidades que puede implicar. Posteriormente, se discuten un conjunto, relativamente idiosincrásico, de temas emergentes dentro de la intervención psicopedagógica. Finalmente, en las conclusiones se intentan apuntar diferentes maneras de dar sentido a todos los temas planteados anteriormente. 
Esta discusión debe servir para comenzar a dar respuesta a dos problemas más amplios dentro de la Psicopedagogía. Por una parte, ofrecer una visión de la Psicopedagogía como campa disciplinar, con su propio ámbito de problemas e instrumentos teóricos. Por otra parte, hacer explícito el tipo de cuestiones que permiten a la Psicopedagogía enfrentarse a los problemas educativos con herramientas propias que van más allá de las contribuciones que se realizan desde las que hasta ahora se consideraban sus disciplinas madre (la Psicología y la Pedagogía).

\section{Los espacios de la psicopedagogía a lo largo del ciclo vital desde una perspectiva contextualista}

La propuesta que presento para organizar los espacios de trabajo de la Psicopedagogía y los problemas teóricos que presentan responde a mi propia orientación teórica y formación disciplinar. Por ello, debe quedar claro que desde otras perspectivas probablemente puedan presentarse otros marcos organizativos igualmente válidos. Dicho esto, mi sugerencia es considerar los diferentes espacios de la Psicopedagogía a lo largo de dos ejes. En primer lugar, las diferentes etapas del ciclo vital, cada una de las cuales tiene unas características específicas y presenta unas posibilidades de actuación diferentes (Baltes, Reese y Lipsitt, 1980). En segundo lugar, los diferentes contextos de desarrollo, ya que los seres humanos a lo largo de nuestras vidas participamos en diferentes espacios sociales y estos cobran una importancia cambiante con el tiempo (Vila, 1998). La figura 1 intenta plasmar la relación entre estos dos ejes y presenta algunos de los espacios más relevantes y que serán objeto de discusión en este trabajo.

Si observamos la figura, que no pretende ser exhaustiva en cuanto a los espacios a señalar, vemos que hay una asociación bastante importante entre cada una de las etapas del desarrollo y los contextos que desempeñan un papel importante a lo largo del ciclo vital. Al nacer la familia es el principal entorno de nuestras vidas y progresivamente este se va ampliando a la escuela y la educación formal, tras la cual nos insertamos en el mundo laboral durante la parte central de nuestras vidas, para posteriormente pasar durante la vejez, una etapa cada vez más prolongada y significativa, a un periodo posterior a la vida laboral activa. Este esquema debe verse como un punto de partida ya que cualquier reflexión sobre él descubre que presenta una serie de simplificaciones que necesitan elaboración.

En primer lugar, es un esquema fuertemente constreñido culturalmente, ya que refleja transiciones y desarrollos propios de una sociedad occidental industrial (post-industrial) y urbana (u orientada a lo urbano). Así, su aplicabilidad en otros contextos socio-culturales o comunidades es bastante limitada. Por ejemplo, las relaciones entre familia y trabajo pueden ser mucho más intimas en otras sociedades. Igualmente, mientras que en nuestra sociedad tanto la etapa de escolarización-instrucción formal y la vejez se están prolongando significativamente, cada uno de los contextos y etapas del ciclo vital puede tener un peso relativo muy diferente en otros contextos culturales e históricos (San Román, 1990; Hutchby y Moran-Ellis, 1998).

En segundo lugar, incluso dentro del sistema social al que mejor se acomoda, la interrelación familia/niñez-educación/juventud-trabajo/adultez-jubilación/vejez es claramente imperfecta. Por un lado, en estas transiciones lo que se señala no es que cada uno de los contextos precedentes desaparezca como espacio de desarrollo (e intervención), sino que desde 
el punto de vista teórico y práctico asumen una primacía diferencial. Así, la familia es un espacio social en el que normalmente participamos de una $\mathrm{u}$ otra manera a lo largo de todo el ciclo vital, aunque su estructura y sentido cambia de modo muy importante en esta trayectoria. Igualmente, aunque estrictamente el trabajo/mundo laboral ocupe un espacio acotado en el tiempo en las etapas centrales de la vida, como contexto tiene relevancia antes y después de desplegarse, en tanto que influye en las decisiones que las personas tenemos que tomar (e.g. elección de estudios, decisiones formativas, etc.) o en la construcción de nuestra identidad incluso después de haber abandonado determinada actividad laboral (cf. Wenger, 1998). Debe señalarse que en esta discusión estamos llamando «trabajo» a cualquier actividad productiva, remunerada o no o tenga lugar dentro o fuera del hogar, donde las personas perciben que despliegan competencias y hacen contribuciones productivas que consideran específicas. Por ejemplo, a lo largo de este siglo en la escuela española rural se preparaba a alumnos y alumnas para ejercer diferentes trabajos fuera de la escuela (i.e. las mujeres en el hogar y los hombres en el campo). Así, en las aulas se proyectaban diferentes trayectorias vitales para hombres y mujeres que el alumnado incorporaba y construía como parte de su identidad a lo largo de su ciclo vital (Poveda, Palomares-Valera y Cano, 2003).

Por otro lado, porque, simultáneamente a los contextos que he señalado, los seres humanos participamos en muchos otros espacios de actividad, de modo voluntario o «involuntario») que pueden tener un impacto importante en nuestro desarrollo educativo y social. A lo largo de la infancia y la juventud, dada la creciente complejidad de nuestro sistema social y las mayores demandas de aprendizaje que se vierten sobre los miembros más jóvenes, se hace patente que el sistema educativo formal no agota todas las necesidades de formación (ver discusión más abajo). Así, existen numerosos «espacios complementarios», como bibliotecas, museos, organizaciones culturales y deportivas, paralelos al sistema educativo en los que participan la infancia y la juventud. Igualmente, muchas familias dedican recursos suplementarios a expandir las aportaciones de la educación formal a través de tutorías privadas y otras actividades formativas complementarias (e.g. clases de informática, de idiomas, de arte, etc.). Todos estos entornos tienen como objetivo optimizar el desarrollo de los más jóvenes y se están convirtiendo en una espacio emergente de intervención psicopedagógica, en tanto que, por una parte, todavía no han recibido el mismo grado de atención como la familia o la escuela y, por otra parte, están mostrando tener un efecto muy importante sobre el desarrollo social y educativo de los estudiantes (Heath, 2001).

Durante la vida adulta y la vejez (y en ocasiones también la infancia y la juventud), los seres humanos participamos en una variedad de contextos institucionales y de servicios que pueden cobrar una importancia vital y, por tanto, ser objeto de intervención psicopedagógica. En el transcurso de nuestras vidas entramos en contacto con el sistema sanitario, el sistema judicial, varias partes de la burocracia estatal, etc. Para la mayor parte de la ciudadanía este contacto es relativamente esporádico e intermitente (o cuando menos, de menor intensidad que el contacto que tenemos con el sistema escolar o laboral), no obstante, las instituciones que he señalado anteriormente forman parte de nuestros derechos sociales y pueden tener un impacto decisivo en nuestras condiciones vitales. Por ello, la formación adecuada de todas las partes implicadas en el proceso puede verse como un área de intervención plenamente justificada. Por ejemplo, en los centros de salud en contextos rurales, el personal de enfermería tiene que asumir y desplegar todo un conjunto de estrategias mediadoras de la alfabetización para poder responder adecuadamente a las demandas y necesidades sanitarias de una población (habitualmente la de mayor edad) que, en muchas ocasio- 
nes, puede considerarse analfabeta (María José Díaz, comunicación personal, 19 de Febrero, 2003). En este caso, para las profesionales implicadas se plantean competencias de carácter educativo para las cuales no están habitualmente preparadas, pero que claramente podrían ser objeto de acción formativa.

Para otras personas este contacto puede ser mucho más prolongado e intenso (e.g. enfermos crónicos, personas institucionalizadas o encarceladas) y, por tanto, las necesidades formativas anteriormente señaladas se hacen más relevantes. Específicamente, desde el marco que estoy proponiendo la intervención con estos colectivos debe contemplar varias de las necesidades y espacios que aparecen en la figura $1 \mathrm{y}$ las transiciones que potencialmente se darán entre ellos (e.g. en el caso de niños enfermos hospitalizados durante periodos largos deben atenderse las necesidades de escolarización; en el caso de personas institucionalizadas -presos, pacientes psiquiátricos- el proceso de «desinstitucionalización» debe contemplar la (re)inserción en el ámbito familiar o laboral; Shethar, 1993; Snow, Burns y Griffin, 1998).

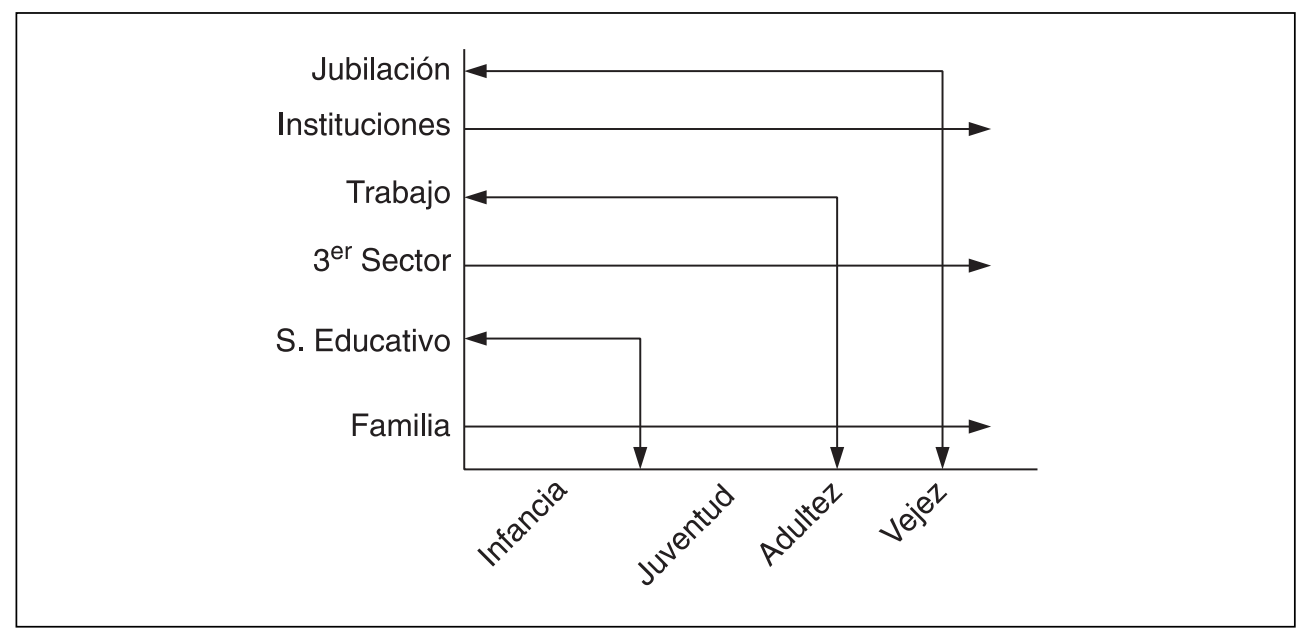

FIGURA 1.

Interrelación entre los contextos de desarrollo y el ciclo vital.

Una vez propuesto el marco, y señaladas algunas puntualizaciones, es posible realizar algunas observaciones sobre el trabajo psicopedagógico. Éste siempre debe tener en cuenta que:

a) los seres humanos participamos en diferentes espacios sociales y, por tanto, las habilidades, competencias y prácticas que desplegamos y adquirimos en un espacio concreto se llevan, con más o menos eficacia y más o menos pertinencia, a otros espacios;

b) que nuestras condiciones vitales en un periodo determinado de nuestra vida están configuradas por nuestra historia previa y proyectan diferentes posibilidades para nuestro desarrollo futuro. Así, una de las competencias centrales del trabajo psicopedagógico debe ser saber considerar e intervenir, desde una perspectiva sincrónica, sobre las diferentes continuidades-discontinuidades entre contextos sociales (Lacasa, 2001) y, desde una perspectiva diacrónica, sobre las diferentes continuidades-discontinuidades entre las etapas del ciclo vital (Neugarten, 1999; Schieffelin, 1987). 


\section{Competencias y desafíos profesionales en la labor psicopedagógica}

El desarrollo de un trabajo profesional dentro del panorama complejo que he presentado implica una serie de desafíos a los que debemos enfrentarnos con un conjunto de actitudes y competencias. La discusión de las funciones de la psicopedagogía suele centrarse en una elaboración de tareas dentro de un marco bien definido (departamento de orientación en un IES, equipo de orientación sectorial, etc.), discutiendo estas tareas, sobre todo, en relación con las funciones asignadas al resto de los profesionales con los que entra en contacto la psicopedagoga (docentes, trabajadoras sociales, inspectoras, directores, etc.). Igualmente, en el plano de las competencias el planteamiento se centra en señalar una serie de habilidades (actitudinales, cognitivas y procedurales) importantes para poder desempeñar el trabajo de forma eficaz.

Esta es una manera importante y fructífera de aproximarse a la cuestión de las tareas y las competencias. Sin embargo, mi aproximación al problema va a ser algo diferente (y quizás no tan operativa), ya que en este epígrafe quiero señalar dos aspectos más transversales en torno la naturaleza del trabajo psicopedagógico. En primer lugar, el tipo de desafíos y consideraciones muy específicas que plantea el marco evolutivo-contextual presentado anteriormente; desafíos que se plasman en la idea de la Psicopedagogía como profesión puente. En segundo lugar, explicitar el modelo de profesional que debe ser asumido por la psicopedagogía: reflexivo.

\section{La psicopedagogía como profesión puente}

El marco presentado anteriormente pone sobre la mesa que el desarrollo integral socioeducativo de un individuo o colectivo va a tener lugar en varios contextos, a lo largo del tiempo y en contacto con varios profesionales. Esta situación implica que el psicopedagogo debe desarrollar una gran capacidad de coordinación (como habilidad cognitiva) entre estos entornos y ser capaz de considerar las necesidades que emergen de este contacto. Esta tarea la recojo bajo la metáfora de construir puentes que, además, es posible formular desde dos puntos de vista: el del receptor de la intervención (que implica «construir puentes entre contextos») y el de las diferentes agentes de intervención (que implica «construir puentes entre profesionales»).

Desde el punto de vista de la persona receptora de la intervención psicopedagógica (la alumna, el cliente, la profesional asesorada, etc.), el trabajo psicopedagógico se realiza en un contexto determinado, pero se espera que las habilidades y estrategias adquiridas en este entorno específico sean transferidas y puestas en práctica en diferentes escenarios sociales. Por ello, es responsabilidad del psicopedagogo ser consciente de las dificultades que pueden estar implicadas en estas transiciones (o continuidades-discontinuidades). Este desafío puede formularse desde dos aproximaciones: por una parte, centrándose en la capacidad reflexiva y contextualista que debe desarrollar la persona receptora de la intervención y, por otra parte, colaborando con los diferentes agentes con un papel educativo con los que entra en contacto la persona receptora del servicio (e.g. maestras, padres, trabajadoras sociales, etc.). Sea cual sea la estrategia de trabajo, el objetivo final es contribuir a que todas las partes implicadas desarrollen una mejor comprensión de las necesidades, intenciones, estrategias y objetivos que tienen el resto de los agentes de cada uno de los contextos educativos contemplados. 
Ahora bien, una parte importante de esta labor de coordinación y construcción de puentes implica considerar las características y expectativas que configuran los contextos principales donde tiene lugar el desarrollo socio-educativo en nuestra sociedad. Por ello, sí que resulta muy importante señalar algunos aspectos que hacen especialmente complejo el trabajo en torno a los dos contextos, cuyo peso antes quise relativizar y que ahora se presentan como ejes de esta labor intercontextual: el escolar y el laboral. Ambos espacios tienen una visibilidad cuantitativa clara y, además, están inmersos en un mar de tensiones a nivel ideológico y político.

En cuanto al sistema educativo formal y la escuela como institución, uno de sus rasgos principales es que todo el conjunto de la sociedad (y no sólo la parte del sistema social implicada directamente como profesional, alumno o familiar) tiene un complejo de ideas y expectativas bastante elaborado sobre sus funciones, organización y objetivos. Esto debe verse como un rasgo estable de la reflexión y el discurso educativo y, en teoría, sus consecuencias no tienen porque ser ni positivas, ni negativas. Sin embargo, sí que quiero señalar dos aspectos que pueden hacer el cambio y la intervención educativa más complicada. Primero, dado este discurso social, cualquier propuesta psicopedagógica va a ser sometida a un escrutinio público muy intenso en el que aparentemente cualquier decisión, independientemente de los criterios técnicos que la guíen, puede ser «contestada» con cierta legitimidad. Esto, en principio, podría ser un impulso para hacer la práctica educativa mucho más transparente y reflexiva, pero hay un segundo rasgo de este escrutinio público que la hace especialmente delicada. Segundo, este discurso público (y esto, aparentemente, se remonta a la Grecia Clásica; Delval, 1997; Berliner y Biddle, 1995), es generalmente peyorativo, en tanto que recurrentemente afirma que los objetivos, prácticas y logros educativos del momento presente son menores que los de las generaciones precedentes $-\mathrm{y}$, además, esta supuesta «degradación» educativa esta a la base de la degeneración social y moral que sufre la juventud de la sociedad. Este es un debate muy apasionado y complejo, en el que se entremezclan aspectos técnicos, ideológicos, económicos y sociales, y en el cual no podemos entrar aquí. Lo importante para los propósitos de este trabajo es señalar cómo éste va a ser, en mayor o menor medida, uno de los elementos que configure el trabajo en contextos escolares y el contacto de la escuela con otras instituciones (familia, trabajo, administración, etc.).

En cuanto al sistema laboral, ya se da por hecho que estamos en un periodo de transformación importante. Además, los cambios y exigencias del sistema laboral y económico emergente aluden a procesos en los que la intervención educativa y psicopedagógica tienen el potencial de desempeñar un papel básico. Antes de discutir estos cambios, la primera cuestión que debe señalarse es que no todos los sectores sociales (definidos en términos geográficos, socio-culturales, políticos, etc.) participan del mismo modo o en el mismo grado en las transformaciones de la «nueva economía». Hecha esta puntualización, hay una serie de rasgos del trabajo en un sistema post-industrial muy relevantes para la planificación de la intervención psicopedagógica y la discusión de las relaciones entre el sistema laboral y otros contextos sociales.

En primer lugar, esta emergiendo como fenómeno estructural que la vida laboral de las trabajadoras va a experimentar muchos más cambios y transformaciones de las que eran habituales en el pasado. La expectativa actual es que los nuevos trabajadores cambien varias veces de puesto, empleo y funciones a lo largo de su vida (y no sólo asciendan en una escala única o desempeñen tareas similares con diferentes empleadores). Lógicamente, esta flexibilidad implica unas necesidades de formación y aprendizaje continuo que no se pro- 
ducían en el pasado y que deben incorporar principios psicopedagógicos nuevos, entre ellos enfrentarse al hecho de que el «conocimiento experto» (cf. Pozo, 1996) se va a convertir en un componente mucho más complejo que incorporará transferencias y bagajes que hasta ahora no se habían producido.

En segundo lugar, las competencias que se requieren para trabajar en la llamada «sociedad de la información», tales como la capacidad para buscar, analizar, gestionar, producir y transmitir conocimientos o la habilidad para trabajar en equipo, comunicarse en diversos medios de modo eficaz y adaptarse a entornos diversos y cambiantes (The New London Group, 1996), hacen referencia a objetivos y prácticas que las Ciencias de la Educación se han planteado y llevan cierto tiempo intentando fomentar (e.g. Cazden, 2001; Baxter, 1999). Si esto es cierto, cuando menos, hay dos puntos que señalar. Por una parte, que las profesionales con una formación inicial en el campo de la educación (como la Psicopedagogía), están en una posición privilegiada para desempeñar un papel clave en la promoción y gestión de estos cambios laborales. Por otra parte, si en el plano del discurso parecen darse unas convergencias importantes entre los objetivos y creencias en el campo educativo y en el campo laboral, esta relación en el plano de la práctica es bastante más compleja. Las relaciones (transiciones, influencias mutuas, etc.) entre el sistema laboral y el sistema educativo no son, ni de lejos, tan fluidas y armónicas. Por tanto, la Psicopedagogía podría ser uno de los campos dedicados a dilucidar las causas de este hiato, reto que puede configurar la investigación y práctica psicopedagógica de los próximos años.

El segundo puente que quiero discutir hace referencia al papel que puede desempeñar una psicopedagoga como dinamizadora y facilitadora dentro de equipos multiprofesionales, independientemente de que sea o no la persona que asuma un puesto de liderazgo, coordinación o dirección -ya que mi objetivo es discutir una capacidad específica, no un puesto de trabajo dentro de una jerarquía. Probablemente, frente a la discusión precedente, esta sea una cuestión algo más específica, pero hace referencia a un aspecto que considero puede ser determinante en la eficacia y gestión del trabajo dentro de equipos, compuestos por varios profesionales de varios campos, o en el trabajo en contacto con profesionales de varias disciplinas (situaciones que se dan cotidianamente en casi todos los entornos en los que se desempeña una labor psicopedagógica).

A lo largo del desarrollo de un trabajo específico es casi seguro que el psicopedagogo va a colaborar o entrar en contacto con diferentes profesionales (maestras, pedagogos, psicólogas, abogados, economistas, trabajadoras sociales, educadores, técnicos específicos, etc.) los cuales tienen un bagaje y una formación específica, igualmente legítima, que permite llegar a soluciones y propuestas de trabajo diferentes. Cada uno estará especialmente cualificado para abordar determinadas tareas e interpretar los problemas desde su propia área experta. En algunas ocasiones estas propuestas son compatibles y en otras entran en conflicto; por ello, gran parte del trabajo en situaciones multiprofesionales implica llegar a acuerdos y establecer consensos compartidos sobre el modo de abordar los problemas y desarrollar estrategias de actuación (e.g. Sánchez, 2000; Crepeau, 2000).

Lograr esto no es en absoluto una tarea sencilla, ya que implica que cada profesional comprometido en este proceso sea capaz de dar respuesta a, al menos, tres cuestiones: (a) qué conocimientos específicos tiene que no comparten sus compañeros; (b) cómo se vinculan estos conocimientos a un cuerpo complejo de saberes formados por las aportaciones de cada profesional; (c) cómo se transmiten de modo claro estos conocimientos a otros profe- 
sionales para que sean incorporados a una propuesta de actuación consensuada. Obviamente, la colaboración interprofesional no siempre se desarrolla de este modo, ni esta es una actitud imprescindible para llevarla a cabo. Dos maneras a través de las que este proceso se puede esquivar son, por una parte, establecer y seguir pautas de trabajo claramente pre-estructuradas y pre-definidas y, por otra parte, seguir una estrategia jerárquica en la que el trabajo conjunto se subordina a las preferencias y saberes de un profesional/campo de conocimientos específicos.

Cada una de estas opciones tiene sus ventajas e inconvenientes y unas posibilidades de aplicación específicas, pero no abordan una gran parte de situaciones laborales en las que los objetivos y los procedimientos del trabajo en equipo se presentan como tareas emergentes que deben ser resueltas sobre la marcha. Bajo estas circunstancias, considero que las tres preguntas que he señalado anteriormente tienen un papel crucial y, además, que la Psicopedagogía es una disciplina especialmente equipada para arrojar luz sobre este proceso. Enfrentarse de modo eficaz a los tres pasos supone ser capaz de desarrollar cierta intersubjetividad profesional y esto implica entrar en el corazón de lo que son procesos de enseñanzaaprendizaje cruciales en el desarrollo de las competencias humanas (Tomasello, 1999a; Tomasello, 1999b; Tomasello, Kruger y Ratner, 1993). Como tarea, requiere desentrañar los conocimientos de cada una de las partes implicadas y desarrollar estrategias para actuar sobre ellas y la Psicopedagogía es una de las disciplinas que tiene estos procesos como cuestión central de su trabajo.

\section{La psicopedagoga como profesional reflexiva}

Si tomamos las diferentes tipologías de formación profesional habituales en las Ciencias de la Educación (e.g. Marchesi y Martín, 1998), creo que es posible afirmar que en la actualidad se ha reavivado el debate entre la visión de un profesional técnico y la visión de un profesional reflexivo. El primer modelo asume que el trabajo implica la aplicación directa de un conocimiento sistematizado y científicamente validado a partir de criterios estrictamente «técnicos». El segundo modelo asume que el trabajo profesional no puede limitarse a esta aplicación, ya que existe un margen amplio de incertidumbre y situaciones no contempladas a las que sólo podemos enfrentarnos reelaborando y reconstruyendo nuestros conocimientos profesionales creativamente a partir de la práctica.

Hasta hace bien poco se daba por hecho que el modelo reflexivo suponía una superación de las limitaciones «obvias» del modelo técnico. Sin embargo, el énfasis renovado en la evaluación en los últimos años de los logros y los resultados de cualquier intervención educativa (accountability) ha hecho que el discurso técnico recupere cierta primacía. Qué implica una posición reflexiva y qué sentido tiene en la práctica educativa son cuestiones ya conocidas dentro de las Ciencias de la Educación, por ello no se van a elaborar más aquí.

Lo que sí que es necesario dejar constatado es que la propuesta que defiendo sobre el trabajo psicopedagógico necesariamente debe incorporar un grado muy alto de reflexividad. Esquemáticamente, esto se apoya en dos rasgos del trabajo educativo. En primer lugar, la naturaleza misma de los problemas educativos, intrínsecamente «mal definidos» (Bruer, 1995), y los conocimientos educativos, multiparadigmáticos y abiertos, hacen que una respuesta estrictamente técnica nunca sea posible. En segundo lugar, la naturaleza política de la práctica y teoría educativa (Luke, 1995; Torres, 1995) implica que cualquier trabajo psi- 
copedagógico también contribuye a avanzar determinada posición ideológica, política y económica. Por ello, la única opción de trabajo es que seamos conscientes y críticos (i.e. reflexivos) del papel social que desempeña cualquier intervención educativa.

\section{Temas emergentes en el trabajo psicopedagógico}

El trabajo en el campo educativo está experimentando una serie de transformaciones importantes que van parejas, por un lado, a los cambios y procesos sociales que se dan en la sociedad y, por otro, a las transformaciones que experimenta el propio sistema educativo en respuesta a estos cambios. En este epígrafe señalaré un conjunto de temas emergentes en el campo psicopedagógico y examinaré el papel que desempeña el marco propuesto en su análisis. Utilizando como heurístico una progresión dentro-fuera (del sistema escolar) estos son:

\section{Contemplar una noción más amplia de la escolarización}

Centrándonos en los objetivos y los recursos movilizados por el propio sistema escolar y dirigidos a la población en edad de escolarización obligatoria (6-16 años), hay una serie de nuevas tareas y espacios susceptibles de ser asumidos por la Psicopedagogía.

En primer lugar, fuertemente impulsados por la reforma educativa, las instituciones educativas cuentan con una serie de servicios de apoyo (para alumnado, familias y docentes), plasmados en departamentos de orientación, equipos sectoriales, etc. que complementan y hacen más eficaz los procesos de enseñanza-aprendizaje en el aula. Este es el espacio tradicional del trabajo psicopedagógico dentro del sistema educativo por lo que no será discutido más aquí. En segundo lugar, el currículum contempla diferentes ejes transversales que permiten incorporar innovaciones psicopedagógicas más fácilmente. Por tanto, es posible que estos ejes se desarrollen a través de estructuras de participación en las que la psicopedagoga desempeñe un papel más activo junto con los docentes y los alumnos.

En tercer lugar, existe cierto consenso de que aprovechar al máximo la educación formal no termina con el trabajo en el aula y desde la escuela se plantean (o deberían plantear) numerosas actividades que implican hacer uso de otros espacios. Como he dicho anteriormente, museos, bibliotecas, granjas escuela, instituciones laborales diversas, etc. son ámbitos en los que las alumnas participan, esporádicamente o de modo más estructurado, para complementar y optimizar los aprendizajes escolares. Igualmente, la escuela se complementa con numerosas actividades extra-escolares, privadas y públicas, con diferentes grados de formalidad, que incluyen de modo significativo (para «remediar» o «optimizar») los resultados escolares. Este conjunto heterogéneo de espacios y problemas suponen un ámbito en el que la Psicopedagogía puede desempeñar un papel muy importante, aunque esto implique formalizar una serie de necesidades educativas que los diferente agentes implicados quizás no tengan plenamente en mente en la actualidad.

\section{Optimización del uso educativo de las nuevas tecnologías}

Las nuevas tecnologías forman parte de las sociedades occidentales post-industriales y desde hace ya algún tiempo viene hablándose del impacto y uso que deben tener en el cam- 
po educativo. También debe darse por hecho que los psicopedagogos deberán incorporarlas como parte de su trabajo más cotidiano. De nuevo nos encontramos con un campo sobre el que se ha escrito y hablado mucho, pero sobre el cual todavía no es fácil establecer algunos puntos de consenso. Aún así, señalaré dos aspectos que creo que deben estar presentes en cualquier aplicación educativa de las nuevas tecnologías y que no siempre son contemplados por profesionales ajenos al campo educativo.

En primer lugar, a pesar de que diseñar y preparar materiales informáticos y multimedia requiere un conocimiento técnico que suele desbordar a la Psicopedagogía, su diseño para fines educativos debe incorporar criterios psicopedagógicos en el proceso. Para que estas herramientas sean educativamente útiles, es imprescindible que, además de ser «atractivas», «divertidas» $\mathrm{y}$ «sencillas», consideren aspectos clave como la secuenciación y estructuración de los contenidos o el tipo de feedback y andamiaje que pueden proporcionar al usuario (cf. Bruer, 1995).

En segundo lugar, además de esta consistencia interna, el diseño de cualquier material informático educativo debe contemplar y tener en consideración en su diseño las situaciones, participantes y estructuras sociales en los que va a ser utilizado. En la mayor parte de los casos el uso de los ordenadores con fines educativos es una situación social en los términos más estrictos, ya que la ratio ordenador/persona casi nunca es $1 / 1$ ni en la escuela, ni en la familia. Por ello, el diseño de software y hardware educativo debe contemplar de modo central la actividad social que se da «con el ordenador, en torno al ordenador y a través del ordenador» (Cazden, 2001). Además, esta valoración de los entornos sociales debe considerar muy seriamente las estructuras sociales más habituales y estables que se dan en espacios como la escuela o la familia independientemente de las nuevas tecnologías. De no hacerlo perderán toda su eficacia ya que, por una parte, no es razonable pensar que la mera incorporación de nuevas tecnologías va a producir por sí cambios radicales en las estructuras sociales y, por otra, una aplicación tecnológica que sea claramente incompatible con la estructura social existente es casi seguro que será desechada (Cole, 1996).

\section{Fomento de la participación comunitaria y la inserción socio-laboral plena}

A pesar del clima neoliberal imperante, los estados continúan asumiendo como una responsabilidad propia el fomento de la igualdad de oportunidades y la participación plena en todos los ámbitos del sistema social de diferentes estamentos poblacionales. Dadas unas condiciones de desigualdad inicial entre diferentes grupos sociales en función de dimensiones como el género (mujeres vs. hombres), la edad (juventud y tercera edad), y sus diferentes problemáticas (discriminación socio-laboral, riesgo de exclusión social, participación activa, etc.), se están desarrollando toda una serie de programas sociales que incorporan objetivos educativos y psicopedagógicos entre sus metas. Los estamentos que desarrollan estos programas (entidades nacionales, supra-nacionales, regionales, locales, públicas y privadas) son muy diversos, pero también se configuran como un ámbito laboral emergente en el que desarrollar una serie de estrategias valiosas por su orientación específicamente psicopedagógica (e.g. Paoletti, 1998; Funes, 2001). 


\section{Incorporar la diversidad cultural como fenómeno estructural}

Los movimientos migratorios, económicos y sociales que han experimentado los países occidentales durante el siglo XX han hecho que la diversidad cultural y étnica se vea como un rasgo básico de los estados-nación actuales. La Psicopedagogía, en todos los ámbitos en los que intervenga, deberá contemplar este hecho como fenómeno estructural y, por ello, diseñar su intervención en función de las implicaciones y necesidades que esto conlleva.

Pensando en el sistema educativo, la bibliografía y reflexión en torno a la diversidad cultural y la educación multi/intercultural ha llegado a ser incuantificable. A pesar de las contradicciones y tensiones que una lectura, por pequeña que sea, de esta bibliografía descubre (Narbona, 1999), casi toda la reflexión en torno a la diversidad cultural en la educación comparte un rasgo común. En estos trabajos se asume, explícitamente o implícitamente, que la educación multi/intercultural se encamina a intervenir sobre una situación de desigualdad entre grupos sociales que de no darse podría derivar en la marginación y exclusión social de un grupo minoritario. Así, la educación inter/multicultural se encamina a mejorar las condiciones educativas de determinado alumnado, pero también para prevenir ciertos costes sociales y personales derivados de la no intervención.

Sin duda, este es el objetivo prioritario y más urgente de la intervención educativa, pero también es importante señalar que la reflexión sobre la diversidad cultural no se limita al sistema escolar y a estas situaciones claramente asimétricas. Un campo emergente, especialmente en entornos laborales, para la intervención psicopedagógica tiene que ver con el trabajo y la comunicación en entornos inter/multiculturales. La globalización económica, la integración europea y el desplazamiento trans-nacional de técnicos y trabajadores cualificados hacen que la diversidad cultural también sea una cuestión central en muchos aspectos del mundo laboral (como los procedimientos de selección de personal, la organización del trabajo en equipo, las políticas de cortesía y convivencia internas a una empresa, etc.). En este caso, los rasgos económicos y laborales de la «población clave» son muy diferentes, pero también implican trabajar bajo el epígrafe de «diversidad cultural» utilizando herramientas psicopedagógicas aunque sea a través de procedimientos distintos (la consultoría, la gestión, etc.) y en entornos muy diferentes a los implícitos en el párrafo anterior (e.g. empresas con un gran poder económico frente a núcleos urbanos degradados).

En conclusión, existen diferentes problemas y ámbitos en los que la Psicopedagogía todavía tiene que desplegar todo su potencial. En España, algunos de estos entornos están relativamente consolidados, otros están en desarrollo y otros todavía debe ser abordados completamente. Los cuatro temas genéricos que he presentado en este epígrafe son una concreción de los espacios sugeridos en el marco contextual y del ciclo vital con el que abrí este escrito. También implican las competencias que he presentado en el epígrafe anterior. Así, a lo largo del trabajo he intentado presentar algunas de las herramientas y retos que presenta el trabajo psicopedagógico. Hecho esto, cabe preguntarse qué problemas y conclusiones pueden derivarse de esta propuesta.

\section{Conclusiones}

El principal problema que emerge de toda la discusión precedente es la interrelación y especificidad de la Psicopedagogía con los diferentes perfiles profesionales que también 
ocupan los ámbitos descritos. Ningún espacio que he descrito esta exclusivamente copado por Licenciados en Psicopedagogía, ya que en estos espacios trabajan igualmente pedagogas, psicólogos, maestras, educadoras sociales o trabajadores sociales, por citar algunos -lista que, como vemos, implica una preparación, en años de formación y contenidos, muy diferente. Además, sobre muchos de los problemas y temas que he presentado como específicamente psicopedagógicos casi todas las disciplinas de la lista anterior podrían hacer la misma apropiación. Esto implica que la Psicopedagogía se inserta en campos «distribuidos» o «disputados» por diferentes profesiones y debe conseguir desarrollar su propia identidad dentro de estas intersecciones.

Dada esta situación, el desarrollo de la formación psicopedagógica en el contexto español presenta una serie de ventajas potenciales, que pueden comenzar a dar respuestas al marco y desafíos presentados a lo largo del trabajo. En primer lugar, el alumnado que conforma a los Licenciados en Psicopedagogía en la mayor parte de los casos procede de otras profesiones (maestros o educadores sociales) o está iniciado en un campo diferente al que conforma su titulación final (Psicología y Pedagogía). Esto implica que, en conjunto, el alumnado tiene una formación más flexible y amplia en cuanto a rango de problemas a considerar que la habitual en otras Licenciaturas. Para la persona que ha completado esta formación implica que puede asumir un número amplio de roles e identidades profesionales a lo largo de su trabajo. Debe resaltarse que la cuestión clave aquí es la identidad profesional que decida asumir el psicopedagogo. Desde disciplinas con una tradición e institucionalización más amplia (como la Psicología o la Pedagogía), la afiliación principal de sus discípulos suele ser con la disciplina, sus cánones teóricos y su identidad académica. Sin embargo, entre los diferentes profesionales de la educación muchas veces el nexo de unión es un interés compartido por una serie de problemas y cuestiones «educativas» (que tienen una vertiente «real», concretada en personas, instituciones, políticas, etc.), más allá de credenciales académicas o bagajes disciplinares. Decantarse por una u otra manera de definir la naturaleza del trabajo que se está llevando a cabo es una tarea por desarrollar, tanto para las psicopedagogas de modo individual como para la Psicopedagogía de modo institucional. Además, la respuesta que se dé a esta cuestión, globalmente o de modo estratégico para problemas o circunstancias específicas, define cómo se van a establecer las conexiones entre contextos, etapas del ciclo vital y espacios profesionales que se han discutido a lo largo del trabajo.

En segundo lugar, formalmente, la Psicopedagogía se define en términos interdisciplinares, teniendo a la Psicología y la Pedagogía como sus referentes principales. No obstante, esta interdisciplinariedad se está convirtiendo en un rasgo esencial de su definición y, cada vez más, la Psicopedagogía incorpora elementos de diferentes disciplinas, además de las anteriores (Economía, Sociología, Antropología, Lingüística, Historia, etc.), para construir su propio lenguaje disciplinar. Además, redundado en el punto señalado anteriormente desde otro ángulo, en el proceso de definición de su campo dentro de las Ciencias Sociales, al contrario de la lista anterior, la Psicopedagogía potencialmente no se define por el rango de aspectos de la experiencia humana que aborda (e.g. la mente, la economía, la cultura, el lenguaje, etc.), sino por centrarse en un conjunto de prácticas y actividades sociales que se definen por tener un carácter educativo. En términos de Manzanares (en prensa), la Psicopedagogía se define por centrarse (investigar e intervenir sobre) estructuras de funcionamiento psicopedagógico, las cuales pueden contemplarse a nivel individual, grupal e institucional. Paralelamente, yo he defendido que deben contemplarse dentro del ciclo vital de los seres humanos y los contextos sociales en los que se ponen en funcionamiento estas estructuras. 


\section{Reconocimientos}

Una versión más amplia de este trabajo fue presentada en el ciclo de conferencias organizado durante Marzo del 2002 por la Facultad de Ciencias de la Educación y Humanidades de la Universidad de Castilla-La Mancha, donde trabajé entre los años 1999-2003, y titulado «El psicopedagogo y sus ámbitos de intervención». Quisiera agradecer los comentarios y sugerencias de Benito del Rincón, Asunción Manzanares, Manuel Palomares-Valera y Ana Cano a versiones previas de este trabajo.

\section{Referencias bibliográficas}

Baltes, P; Resse, H. y Lipsitt, L. (1980): «Life-span developmental psychology». Annual Review of Psychology, 31, 165-110.

Baxter, J. A. (1999): «Teaching girls to speak out: The female voice in public contexts«. Language and Education, 13 (2), 81-98.

Berliner, D. y Biddle, B. (1995): The manufactured crisis: Myths, fraud and the attack on America's public schools. Nueva York: Addison-Wesley.

Bruer, J. (1995): Escuelas para pensar: Una ciencia del aprendizaje en el aula. Barcelona: Paidós.

Cazden, C. (2001): Classroom discourse ( $2^{\text {nd }}$ edition). Portsmouth: Heinneman/

Cole, M. (1996): Cultural psychology: A once and future discipline. Cambridge: Cambridge University Press.

Crepeau, E. (2000): «Reconstructing Gloria: A narrative analysis of team meetings». Qualitative Health Research, 10 (6), 766-788.

Delval, J. (1996): Los fines de la educación (3 $3^{\text {a }}$ edición). Madrid: Siglo XXI.

Funes, J. (2001): «Dificultades de adaptación social en la adolescencia». En. B. del Rincón (coord.), Presente y futuro del trabajo psicopedagógico (pp. 159-178). Barcelona: Ariel.

Heath, S. B. (2001): «Three's not a crowd: Plans, roles, and focus in the Arts». Educational Researcher, 30 (7), 10-17

Hutchby, I. y Moran-Ellis, J. (coords.) (1998): Children and social competence: Arenas of action. Londres: Falmer Press.

Lacasa, P. (2001): «Entorno familiar y educación escolar: la intersección de dos escenarios educativos». En C. Coll, J. Palacios y A. Marchesi (comps.), Desarrollo psicológico y educación 2: Psicología de la educación escolar (pp. 597-632). Madrid: Alianza.

Luke, A. (1995): «Text and discourse: An introduction to Critical Discourse Analysis». Review of Research in Education, 21, 3-48.

Manzanares, A. (en prensa): «Competencias del psicopedagogo: Una visión integradora de los espacios de actuación». Bordón.

Marchesi, A. y Martín, A. (1998): Calidad de la enseñanza en tiempos de cambio. Madrid: Alianza.

Narbona, L. (1999): «Elementos críticos y propuestas para eliminar tópicos: Marroquíes, migración y escuela». En M. A. Essomba (coord.), Construir la escuela intercultural: Reflexiones y propuestas para trabajar la diversidad étnica y cultural (pp. 173-184). Barcelona: Grao

Neugarten, B. (1999): Los significados de la edad. Barcelona: Herder.

Paoletti, I. (1998): Being an older woman: A study in the social production of identity. Londres: Lawrence Erlbaum Associates. 
Poveda, D.; Palomares-Valera, M. y Cano, A. (2003): «Putting school in its place: A narrative analysis of the educational memories of adult and elder people». Póster presentado en 2003 AERA (American Educational Research Association) Annual Meeting, Chicago, 21-25 de Abril.

Pozo, I. (1996): Aprendices y maestros: La nueva cultura del aprendizaje. Madrid: Alianza.

Rockwell, E. (1995): «De huellas, bardas y veredas». En E. Rockwell (coord.), La escuela cotidiana (pp. 13-57). México D.F.: Fondo de Cultura Económica.

Sánchez, E. (2000): «El asesoramiento psicopedagógico: un estudio observacional sobre las dificultades de los psicopedagogos para trabajar con los profesores». Infancia y Aprendizaje, 91, 55-77.

San Román, T. (1990): Vejez y cultura: Hacia los límites del sistema. Barcelona: Fundación La Caixa.

Schieffelin, B. (1987): «Do different worlds mean different words: an example from Papua New Guinea». En S. Phillips, S. Steele y C. Tanz (coords.), Language, gender and sex in comparative perspective (pp. 249-260). Cambridge: Cambridge University Press.

Shethar, A. (1993): «Literacy and "empowerment"? A case study of literacy behind bars». Anthropology and Education Quarterly, 24 (4), 357-372.

Snow, C; Burns, M.S. y Griffin, P. (coords.) (1998): Preventing reading difficulties in young children. Washington D.C: National Academy Press.

The New London Group (1996): «A pedagogy of multiliteracies: Designing social futures». Harvard Educational Review, 66, 60-92.

Tomasello, M. (1999a): «The human adaptation for culture». Anoual Review of Anthropology, 28, 509-529.

Tomasello, M. (1999b): The cultural origins of human cognition. Cambridge: Cambridge University Press.

Tomasello, M.; Kruger, A.C. y Ratner, H. (1993): «Cultural learning». Behavioral and Brain Sciences, 16, 495-552.

Torres, C. (1995): «State and education revisited: Why educational researchers should think politically about education». Review of Research in Education, 21, 255-331.

Vila, I. (1998): Familia, escuela, comunidad. Barcelona: Horsori.

Wenger, E. (1998): Communities of practice: Learning, meaning, and identity. Cambridge: Cambridge University Press.

Willis, P. (1993): «Producción cultural no es lo mismo que Reproducción Cultural, que a su vez no es lo mismo que Reproducción Social, que tampoco es lo mismo que Reproducción». En H. Velasco, F. García y A. Díaz de Rada (eds.), Lecturas de antropología para educadores (pp. 431-461). Madrid: Trotta.

Fecha de recepción: 13-05-02

Fecha de revisión: 16-06-03

Fecha de aceptación: 27-10-03 